\title{
QUASI-ATOMS AND COMPLETE DISTRIBUTIVITY
}

\author{
ZIKE DENG
}

\author{
(Communicated by William C. Waterhouse)
}

\begin{abstract}
We introduce quasi-atoms in completely distributive complete lattices and use them to treat the representation of these lattices and their connection with a binary relation.
\end{abstract}

1. Introduction. McKinsey and Tarski extended the frame of topological spaces to Boolean lattices in their algebra of topology [5], of which the local properties were treated by means of atoms in [6]. On the other hand fuzzy topology is but a special case of the generalized algebra of topology, i.e., the topology of algebra with polarity instead of Boolean complement and its local properties can be treated by means of fuzzy points such as in [7]. The quasi-atoms and the binary relation $\prec$ in this paper seem to be the ideal tools of treating the local properties of these generalized algebras, which unify the ways in treating [6 and 7] respectively.

Some ideas and techniques in this paper develop those of [7] and overlap those of $[8,9,10,11$ and 12].

We first introduce quasi-atoms in completely distributive complete lattices, which are generalization of atoms and completely $V$-irreducible elements. And then we introduce a base and give a procedure for generating these lattices by it. Finally we formulate the representation of these lattices by a certain class of complete $\cup$-semirings and their connection with a binary relation.

2. Quasi-atoms. Throughout this paper $(L, \leq)$ will denote a completely distributive complete lattice. Its elements will be denoted by $a, b, c$ and its subsets by $A, B, C$.

Definition $2.1[\mathbf{1}] . a \prec b$ iff for every $A$ with $\bigvee A=b$ there exists $c \in A$ such that $a \leq c$.

Now we summarize the properties of the binary relation $\prec$ which will be used frequently in the following lemma.

LEMMA 2.1 [1, LEMMA 2.3]. (1) $a \prec b$ implies $a \leq b$,

(2) $a \leq b \prec c$ implies $a \prec c$,

(3) $a \prec b$ implies that there exists $c$ such that $a \prec c \prec b$,

(4) $a \prec a$ iff $a$ is completely $\vee$-irreducible,

(5) $a \prec \bigvee A$ iff there exists $b \in A$ such that $a \prec b$,

(6) $a=\bigvee_{b \prec a} b$.

DEFINITION 2.2. Let $\Delta(L)=\left\{\bigwedge_{a \prec b} b \mid a \neq 0, \bigvee_{a \ddagger c} c<1\right\}, p \in \Delta(L)$ is called a quasi-atom.

Received by the editors November 3, 1986 and, in revised form, April 23, 1987.

1980 Mathematics Subject Classification (1985 Revision). Primary 06D05, 06D10.

Key words and phrases. Quasi-atoms, bases, complete U-semirings, pseudo orderings. 
We use $p, q, r$ to denote elements of $\Delta(L)$ and $P, Q, R$ its subsets. Let $I(L)$ be the set of all completely $\vee$-irreducible elements of $L$ and $A(L)$ the set of all its atoms, then it is evident that $A(L) \subseteq I(L) \subseteq \Delta(L)$.

PROPOSITION 2.1. $P=\bigwedge_{p \prec a} a$.

PROOF. Let $p=\bigwedge_{b \prec c} c$, then $b \prec c$ implies that there exists $d \in L$ such that $b \prec d \prec c$, hence $p \leq d$ and so $p \prec c$. It follows that $\bigwedge_{p \prec a} a \leq \bigwedge_{b \prec c} c$, which implies $p=\bigwedge_{p \prec a} a$. Q.E.D.

PROPOSITION 2.2. $a=\bigvee_{p \prec a} p$.

ProOF. Suppose $a \neq 0$ and $0 \neq b \prec a$. Put $p_{b}=\bigwedge_{b \prec c} c$, then $b \leq p_{b}$. Since $a \not \bigvee_{b \ddagger d} d, p_{b} \in \Delta(L) . b \prec c \prec a$ for some $c$ and $p_{b} \leq c$, so $p_{b} \prec a$. Consequently $a \leq \bigvee_{p \prec a} p$ and so $a=\bigvee_{p \prec a} p$. Q.E.D.

\section{Bases.}

DEFINITION 3.1. $Q \subseteq \Delta(L)$ is called a base of $(L, \leq)$ iff $a=\bigvee\{p \mid p \prec a, p \in Q\}$ for every $a \in L$.

Proposition 2.2 implies immediately the following proposition.

PROPOSITION 3.1. Every $(L, \leq)$ has a base.

DEFINITION 3.2. $p \in \Delta(L)$ is said to have the $\wedge$-property iff for every $a$ and $b$, $p \prec a$ and $p \prec b$ implies $p \prec a \wedge b$.

DEFINITION 3.3. A standard base (in short, $s$-base) is a base of which every element is $\vee$-irreducible and has the $\wedge$-property.

DEFINITION 3.4. $(L, \leq)$ is said to be standard iff it has an $s$-base.

PROPOSITION 3.2. (1) If $(L, \leq)$ is isomorphic to the direct product of a family of complete chains, then it is standard.

(2) If $(L, \leq)$ is isomorphic to a complete ring of sets, then it has the s-base $I(L)$ which is also the minimum of all bases.

(3) If $(L, \leq)$ is atomic, then it has the s-base $A(L)$ which is also the unique base.

PROOF. (1) Being totally ordered, a complete chain is standard. Let $L=\pi L_{i}$ where $L_{i}$ is a complete chain for $i \in I$, then $\Delta(L)$ is an $s$-base. To see this it is enough to note that $\Delta(L)$ consists of those elements of $L$ which are of the form $q^{i}$ for some $i \in I$ where $q^{i}(i) \in \Delta\left(L_{i}\right)$ and $q^{i}(j)=0_{j}$ for $j \neq i$, and that $q^{i} \prec \alpha \in L$ iff $q^{i}(i) \prec_{i} a(i)$.

(2) Since $p \prec a$ iff $p \leq a$ for every $p \in I(L), I(L)$ is an $s$-base by [2, Theorem 2]. Suppose $Q$ is a base, then $I(L) \subseteq Q$ by complete $V$-irreducibility.

(3) $A(L)$ is evidently an $s$-base. Suppose $Q \subseteq \Delta(L)$ is any base. Let $p \in A(L)$, then $q \prec p$ for some $q \in Q$, hence $p=q \in Q$. Thus $A(L) \subseteq Q$. On the other hand let $q \in Q$. Then $q \prec a$ for some $a \in L$. Since $a=\bigvee\{p \mid p \leq a, p \in A(L)\}$, there exists $p \in A(L)$ with $p \leq a$ such that $q \leq p$, hence $q=p \in A(L)$. Therefore $Q \subseteq A(L)$. From above it follows $Q=A(L)$, and so $A(L)$ is a unique base. Q.E.D.

In the remaining part of this section $P \subseteq \Delta(L)$ will be a base. 
DEFINITION 3.5. $R \subseteq P$ is called an $l$-set iff it satisfies the condition:

(1) $p \in R$ implies that there exists $q \in R$ such that $p \prec q$,

(2) $p \prec q \in R$ implies $p \in R$ for every $p \in P$.

The set of all $l$-sets of $P$ is denoted by $L(P)$. For $a \in L, R_{a}=\{p \mid p \prec a$, $p \in P\} \in L(P)$. Let $\Phi: P \rightarrow L(P)$ be defined as $\Phi(q)=R_{q}$ and $\prec_{P}$ the restriction of $\prec$ on $P$.

LEMMA 3.1. $p \prec \bigvee Q$ iff $p \in Q$ for every $Q \in L(P)$ and $p \in P$.

PROOF. By Lemma 2.1(5) and the construction of $Q$. Q.E.D.

By the definition of a base and Lemma 2.1 the following lemma holds.

LEMMA 3.2. (1) For every $p \in P, p \prec a$ implies $p \prec q \prec a$ for some $q \in P$.

(2) $a \leq b$ iff for every $p \in P, p \prec a$ implies $p \prec b$.

(3) For every $p \in P, p \prec \bigwedge A$ iff there exists $q \in P$ such that $p \prec q$ and $q \prec a$ for every $a \in A$.

(4) For every $p \in P, p=\bigwedge\{q \mid q \in P, p \prec q\}$.

PROPOSITION 3.3. (1) $\prec_{p} \circ \prec_{p}=\prec_{p}$.

(2) $q \prec r \in \bigcap_{p \prec s} R_{s}$ implies $q \prec p$ for every $p, q, r, s \in P$.

(3) For every $p \in P$ there exists $q, r \in P$ such that $q \prec p \prec r$.

(4) $R_{p}=R_{q}$ implies $p=q$ for every $p, q \in P$.

(5) $R_{p} \subseteq R_{q}$ and $q \prec r$ imply $p \prec r$ for every $p, q, r \in P$.

If $P$ is an $s$-base, then

(6) $p \in R_{q} \cap R_{r}$ implies there exists $s \in P$ such that $p \prec s$ and $s \in R_{q} \cap R_{r}$ for every $p, q, r \in P$.

(7) For every $Q, S \in L(P)$ and $p \in P, R_{p}=Q \cup S$ implies $R_{p}=Q$ or $R_{p}=S$. If $P=I(L)$, then

(8) $\prec_{P}$ is a partial ordering.

If $P=A(L)$, then

(9) $\prec_{P}$ is the same as the identity relation.

PROOF. (1) follows from Lemma 2.1(1), (2) and Lemma 3.2(1).

(2) follows from Lemma 3.2(2), (3), (4).

(3) That for every $p \in P$ there exists $r \in P$ such that $r \prec p$ is trivial. Let $p=\bigwedge_{b \prec d} d$ and $a_{b}=\bigvee_{b \nsubseteq c} c$. Since $1 \npreceq a_{b}, b \prec 1$, which implies $b \prec c \prec 1$ for some c. Thus $p \leq c$ and so $p \prec 1$. By Lemma 3.2(1) $p \prec q \prec 1$ for some $q \in P$.

(4) follows from Lemma 3.2(2).

(5) follows from Lemma 3.2(2).

(6) follows from the $\wedge$-property of $p \in P$ and Lemma 3.2(3).

(7) follows from the $\vee$-irreducibility of $p \in P$, Lemma 3.1 and Lemma 3.2(2).

(8) follows from Lemma 2.1(4).

(9) is trivial. Q.E.D.

4. Generation of $(L, \leq)$ by a base. In the following proposition suppose $P \neq \varnothing$ is any set and $\prec$ any binary relation defined on it. We shall use $p, q, r, s$ to denote elements of $P$. Let $L(P), R_{p}$ and $\Phi: P \rightarrow L(P)$ be defined as in or below Definition 3.5. We shall use $Q, R, S$ to denote elements of $L(P)$. For brevity by $P_{i, j}, \ldots$ is meant $(P, \prec)$ satisfying the conditions of $(i),(j), \ldots$ in Proposition 3.3 where we need to write $\prec$ instead of $\prec_{p}$ in condition (1). 
PROPOSITION 4.1. (1) $l\left(P_{1,2}\right)$ ordered by inclusion is a completely distributive complete lattice, $\Phi\left(P_{1,2}\right)-\{0\}$ is a base and $\Phi$ is $\prec-p r e s e r v i n g$.

(2) $\Phi\left(P_{1,2,3}\right)=\Phi\left(P_{1,2,3}\right)-\{0\} \neq \varnothing$.

(3) $\Phi: P_{1,2,4} \rightarrow \Phi\left(P_{1,2,4}\right)$ is an injection.

(4) $\Phi^{-1}: \Phi\left(P_{1,2,4,5}\right) \rightarrow P_{1,2,4,5}$ is $\prec$-preserving.

(5) $R_{p} \in \Phi\left(P_{1,2,5,6}\right)$ has the $\wedge$-property.

(6) $R_{p} \in \Phi\left(P_{1,2,7}\right)$ is $\vee$-irreducible.

(7) $\Phi\left(P_{8}\right)$ is the s-base $I\left(L\left(P_{8}\right)\right)$.

(8) $\Phi\left(P_{9}\right)$ is the s-base $A\left(L\left(P_{9}\right)\right)$.

Proof. (1) Since $\bigcup S_{i} \in L\left(P_{1,2}\right), \bigvee S_{i}=\bigcup S_{i}$ and so $L\left(P_{1,2}\right)$ is complete. Furthermore $p \in S$ is equivalent to $p \prec q \in S$ for some $q$, which is equivalent to $p \in$ $\bigvee_{q \in S} R_{q}$, it follows that $S=\bigvee_{q \in S} R_{q}$. It can be easily verified that $q \in S$ implies $R_{q} \prec S$. Thus a fortiori $S=\bigvee_{R \prec S} R$, from this follows complete distributivity by [3, Theorem 1]. On the other hand $\bigwedge S_{i}=\{p \mid$ there exists $q$ such that $p \prec q$ and $q \in S_{i}$ for every $\left.i\right\}$, we have $R_{p}=\bigwedge_{p \in R_{q}} R_{q}$ and so $R_{p}=\bigwedge_{R_{p} \prec S} S$, which implies $\Phi\left(P_{1,2}\right)-\{0\}$ is a base. Finally $\Phi$ is $\prec$-preserving, for $p \prec q$ implies $p \in R_{q}$, which implies $R_{p} \prec R_{q}$.

(2) Since for every $q$ there exists $p$ such that $p \prec q$, it follows that $0 \neq P_{1,2,3}=$ $1 \in L\left(P_{1,2,3}\right)$. Furthermore $P_{1,2,3}=\bigwedge_{p \in P_{1,2,3}} R_{p}$, hence there exists $p$ such that $R_{p} \neq 0$, which implies $\Phi\left(P_{1,2,3}\right)-\{0\} \neq \varnothing$. Since for every $q$ there exists $p$ such that $p \prec q$, it follows that $R_{q} \neq 0$ in $\Phi\left(P_{1,2,3}\right)$ and so $\Phi\left(P_{1,2,3}\right)=\Phi\left(P_{1,2,3}\right)-\{0\}$.

(3) is trivial.

(4) If $R_{p} \prec Q$ in $L\left(P_{1,2,4,5}\right)$, then there exists $q \in Q$ such that $R_{p} \leq R_{q}$, hence $q \prec r \in Q$ for some $r \in Q$, which implies $p \prec r$ and so $p \in Q$. Thus $R_{p} \prec R_{q}$ implies $p \in R_{q}$, which implies $p \prec q$.

(5) If $R_{p} \prec R_{q}$ and $R_{p} \prec R_{s}$, then $p \in R_{q} \cap R_{s}$, hence $p \prec r$ and $r \in R_{q} \cap R_{s}$ for some $r$, which implies $p \in R_{q} \wedge R_{s}$ and so $R_{p} \prec R_{q} \wedge R_{s}$. From this follows the $\wedge$-property by noting that $\Phi\left(P_{1,2,5,6}\right)-\{0\}$ is a base.

(6) is trivial.

(7) $P_{8}$ satisfies automatically the conditions (1)-(5) in Proposition 3.3, so $\Phi\left(P_{8}\right)$ is a base. Since $p \prec p$, it follows that $R_{p} \prec R_{p}$ and so $R_{p} \in I\left(L\left(P_{8}\right)\right)$. Thus $\Phi\left(P_{8}\right)=I\left(L\left(P_{8}\right)\right)$, for $I\left(L\left(P_{8}\right)\right)$ is the minimum base.

(8) As in (7) $\Phi\left(P_{9}\right)$ is also a base. Since $R_{p}=\{p\}, R_{p} \in A\left(L\left(P_{9}\right)\right)$. Thus $\Phi\left(P_{8}\right)=A\left(L\left(P_{9}\right)\right)$, for $A\left(L\left(P_{9}\right)\right)$ is the unique base. Q.E.D.

The above proposition can be used to construct new completely distributive complete lattices from old ones.

LEMMA 4.1. If $P$ is a base of $(L, \leq)$, then $L(P)$ is isomorphic to $(L, \leq)$.

Proof. Define $\psi: L(P) \rightarrow L$ and $\psi(Q)=\bigvee Q$. Since $R_{a} \in L(P)$ for every $a \in L, \psi$ is onto. By Lemma $3.1 \psi$ is one-to-one and $Q \leq R$ implies and is implied by $\psi(Q) \leq \psi(R)$. Q.E.D.

5. Representation of $(L, \leq)$. Let $X \neq \varnothing$ be any set and $P(X)$ the power set. DEFINITION 5.1. (1) A complete $U$-semiring is a family $\tau \subseteq P(X)$ which is closed under arbitrary unions.

(2) For $M \in P(X)$ and $N \in \tau, M^{0}=\bigcup\{N \mid N \subseteq M\}$.

(3) For $N \in \tau$ and $x \in X, M_{x}=M^{0}$ where $M=\bigcap\{N \mid x \in N\}$. 
A complete $U$-semiring ordered by inclusion is a complete lattice. Let $\rho(X)$ be the set of those complete $U$-semirings satisfying the condition (1) $N \leq \bigcup_{x \in N} M_{x}$, $\rho^{*}(X)$ the subset of $\rho(X)$ of which every element is a ring of sets and satisfies the condition (2) $M_{x}$ is $\vee$-irreducible and the condition (3) that $M_{x} \subseteq M_{y}$ and $y \in N$ implies $x \in N$. Finally the set of all complete rings of sets is dentoed by $\rho^{* *}(X)$. Since for $\tau \in \rho^{* *}(X), M_{x}=\bigcap\{N \mid x \in N\}, M_{x}$ is completely $\vee$-irreducible and the above conditions (1), (2), (3) are satisfied automatically, it follows that $\rho^{* *}(X) \subseteq \rho^{*}(X)$.

THEOREM 5.1. (1) A complete lattice is completely distributive iff it is isomorphic to $\tau \in \rho(X)$ for some $X$.

(2) A completely distributive complete lattice is standard iff it is isomorphic to $\tau \in \rho^{*}(X)$ for some $X$.

(3) A completely distributive complete lattice has an s-base consisting of all completely $\vee$-irreducible elements iff it is isomorphic to $\tau \in \rho^{* *}(X)$ for some $X$.

(4) A completely distributive complete lattice has an $s$-base consisting of all atoms iff it is isomorphic to $P(X)$ for some $X$.

Proof. (1) Necessity. Choose $X=P$, where $P$ is a base of $(L, \leq)$. By Lemma $4.1 L$ is isomorphic to $L(P)$. From Propositions 3.3 and 4.1 we see that $L(P)$ is a complete $U$-semiring and $R_{p}=\bigwedge_{R_{p} \prec S_{i}} S_{i}=\bigcup_{T_{j} \subseteq \cap S_{i}} T_{j}$, which implies $R_{p}=M_{p}$ and so $N=\bigcup_{p \in N} M_{p}$. Thus $L(P) \in \rho(P)$.

SUFFICIENCY. Let $\tau \in \rho(X)$. It can be easily shown that $x \in N$ implies $M_{x} \prec N$. By hypothesis $N \leq \bigcup_{x \in N} M_{x}$, a fortiori $N \leq \bigvee_{M \prec N} M$, which implies complete distributivity by $[\mathbf{3}$, Theorem $\mathbf{1}]$.

(2) Necessity. From (1) we see that $L$ is isomorphic to $L(P) \in \rho(P)$. From Propositions 3.3 and 4.1 it follows that $L(P) \in \rho^{*}(P)$.

SUFFICIENCY. From (1) we see that $\tau \in \rho^{*}(X)$ is completely distributive. It can be easily shown that $\left\{M_{x} \mid M_{x} \neq \varnothing, x \in X\right\}$ is an $s$-base.

(3) follows from Proposition 3.2(2) and [2, Theorem 2].

(4) follows from Proposition 3.3. Q.E.D.

6. A binary relation. Let $\rho(X), \rho^{*}(X)$ and $\rho^{* *}(X)$ be defined as in $\S 5$ and let $L(X, \prec)$, the set of all $l$-sets of $X$ with a binary relation $\prec$ be defined as in $\S 3$.

DEFINITION 6.1. (1) A binary relation $\prec$ is said to be $w$-dense iff $x \prec y$ implies that $x \prec z \prec y$ for some $z$.

(2) $\prec$ is said to be $s$-dense iff $x \prec y$ and $x \prec z$ implies that there exists $w$ such that $x \prec w \prec y$ and $x \prec w \prec z$.

(3) $\prec$ is said to be $s$-transitive iff for every $z, z \prec x$ implies $z \prec y$ and $y \prec w$ implies $x \prec w$.

(4) $\prec$ is called a pseudo ordering iff it is transitive and $w$-dense.

$(5) \prec$ is called an $s$-pseudo ordering iff it is $s$-dense and $s$-transitive and satisfies $R_{x}=\{y \mid y \prec x\}$ is $\vee$-irreducible in $L(X)$.

$(6) \prec$ is called a quasi ordering iff it is a pseudo ordering and reflexive.

Evidently a binary relation is a quasi ordering implies that it is an $s$-pseudo ordering, which implies that it is a pseudo ordering.

Let $\theta(X)$ be the set of all pseudo orderings on $X, \theta^{*}(X)$ the set of all $s$-pseudo orderings on $X$ and $\theta^{* *}(X)$ the set of all quasi orderings on $X$, then $\theta^{* *}(X) \subseteq$ $\theta^{*}(X) \subseteq \theta(X)$. The following theorem generalizes the result in [4, Theorem 1]. 
THEOREM 6.1. (1) There is a bijection from $\rho(X)$ onto $\theta(X)$.

(2) There is a bijection from $\rho^{*}(X)$ onto $\theta^{*}(X)$.

(3) There is a bijection from $\rho^{* *}(X)$ onto $\theta^{* *}(X)$.

ProOF. (1) For $\tau \in \rho(X)$ define a binary relation $\lambda(\tau)$ on $X$ as follows: $x \lambda(\tau) y$ iff $x \in M_{y} \in \tau$. It can be easily verified that $\lambda(\tau) \in \theta(X)$ and $\tau=L(X, \lambda(\tau))$. Thus $\lambda: \rho(X) \rightarrow \theta(X)$ is one-to-one. Furthermore since for a binary relation $\prec \in \theta(X)$, $L(X, \prec) \in \rho(X)$ and $\lambda(L(X, \prec))$ is the same as $\prec$, it follows that $\lambda$ is onto.

(2) Since $\tau \in \rho^{*}(X)$ is equivalent to $\lambda(\tau) \in \theta^{*}(X)$, the restriction of $\lambda$ on $\rho^{*}(X): \rho^{*}(X) \rightarrow \theta^{*}(X)$ is a bijection.

(3) Since $\tau \in \rho^{* *}(X)$ is equivalent to $\lambda(\tau) \in \theta^{* *}(X)$, the restriction of $\lambda$ on $\rho^{* *}(X): \rho^{* *}(X) \rightarrow \theta^{* *}(X)$ is a bijection. Q.E.D.

\section{REFERENCES}

1. Ph. Dwinger, Characterization of the complete homomorphic images of a completely distributive complete lattice. I, Nederl. Akad. Wetensch. Indag. Math. 44 (1982), 403-414.

2. G. N. Raney, Completely distributive complete lattices, Proc. Amer. Math. Soc. 3 (1952), 667680.

3. __ A subdirect-union representation for completely distributive complete lattices, Proc. Amer. Math. Soc. 4 (1953), 518-522.

4. G. Birkhoff, On rings of sets, Duke Math. J. 3 (1937), 443-454.

5. J. C. C. McKinsey and A. Tarski, The algebra of topology, Ann. of Math. (2) 45 (1944), 141-191.

6. G. Nöbeling, Grundlagen der analytischen Topologie, Springer-Verlag, Berlin and New York, 1954.

7. Z. Deng, Neighborhoods in fuzzy topological spaces, J. Hunan Univ. 7 (1980), 1-11.

8. J. B. Wright, E. G. Wagner and J. M. Thatcher, $A$ uniform approach to inductive posests and inductive closure, Theoretical Computer Science 7 (1978), 57-77.

9. H. J. Bandelt and M. Erné, The category of z-continuous posets, J. Pure Appl. Algebra 30 (1983), 219-226.

10. D. Novak, Generalization of continuous posets, Trans. Amer. Math. Soc. 272 (1982), 645-667.

11. J. D. Lawson, The duality of continuous posets, Houston J. Math. 5 (1979), 357-394.

12. K. H. Hoffman, Continuous posets and adjoint sequences, Semigroup Forum 18 (1979), 173188.

Department of Applied Mathematics, Hunan University, Changsha, hunan, CHINA 